\title{
Dirac-Kähler approach connected to quantum mechanics in Grassmann space
}

\author{
N. Mankoč Borštnik \\ Department of Physics, University of Ljubljana, Jadranska 19, Ljubljana, Solvenia, \\ J. Stefan Institute, Jamova 39, Ljubljana, 1111, Slovenia, \\ and Promфrska Institute for Natural Sciences and Technology, Marežganskega upфra 2, Koper, 6000, Slovenia
}

H. B. Nielsen

Department of Physics, Niels Bohr Institute, Blegdamsvej 17, Copenhagen, DK-2100, Denmark

and TH Division, CERN, CH-1211 Geneva 23, Switzerland

(Received 27 September 1999; published 18 July 2000)

\begin{abstract}
We compare the way one of us obtained spinors from fields, which are a priori antisymmetric tensor fields, to the Dirac-Kähler rewriting. Since using our Grassmann formulation is simple it may be useful in describing the Dirac-Kähler formulation of spinors and in generalizing it to vector internal degrees of freedom and to charges. The "cheat" concerning the Lorentz transformations for spinors is the same in both cases and is put forward in the Grassmann formulation. Also the generalizations are clearly pointed out. The discrete symmetries are discussed, in particular the appearance of two kinds of time-reversal operators as well as the unavoidability of four families and the evenness and oddness of the Dirac matrices.

PACS number(s): 04.50. $+\mathrm{h}, 11.10 . \mathrm{Kk}, 11.30 .-\mathrm{j}, 12.10 .-\mathrm{g}$
\end{abstract}

\section{INTRODUCTION}

Kähler [1] has shown how to pack the Dirac wave function into the language of differential forms, in the sense that the Dirac equation is an equation in which a linear operator acts on a linear combination $u$ of $p$ forms $(p=0,1, \ldots, d$; here $d=$ dimension $=4)$. This is the Dirac-Kähler formalism.

One of us [2-4] developed long ago an a priori rather different formalism in an attempt to unify spin and charges. In this approach the spin degrees of freedom come out of canonically quantizing certain Grassmannian odd variables $\theta^{a}$ (position analogue in the sense of being on an analogue footing with $x^{a}$ ). These variables are denoted by a vector index $a$, and there are at first no spinors at all to see.

One of the main purposes of the present article is to point out the analogy and nice relations between the two different ways of achieving the-almost miraculous - appearance of spin-one-half degrees of freedom in spite of starting from pure vectors and tensors.

Of course it is a priori impossible that vectorial and tensorial fields (or degrees of freedom) can be converted into spinorial ones without some "cheating." The "cheat" consists really of exchanging one set of Lorentz transformation generators for another set (which indeed means putting strongly to zero one type of Grassmann odd operators fulfilling the Clifford algebra and anticommuting with another type of Grassmann odd operators, which also fulfill the Clifford algebra [2-4]).

In fact, one finds on page 512 in Kähler's article [1] that there are two sets of rotation generators: one set for which the $u$ field (in Kähler's notation) transforms as a spinor field and another one for which it transforms as superpositions of vector and (antisymmetric) tensor fields. Analogously, in the approach of one of us, the a priori Lorentz transformation generators $\mathcal{S}^{a b}=\widetilde{S}^{a b}+\widetilde{\widetilde{S}}^{a b}$ have the wave function transform as vectors and antisymmetric tensors, while $\widetilde{S}^{a b}\left(=-i \frac{1}{4}\left[\widetilde{a}^{a}, \widetilde{a}^{b}\right]\right)$ or $\widetilde{\widetilde{S}}^{a b}\left(=-i \frac{1}{4}\left[\widetilde{\widetilde{a}}^{a}, \widetilde{\widetilde{a}}^{b}\right]\right.$ and $[$,$] means the commutator) used alone are also possible$ Lorentz generators for which the wave function now transforms as a spinor wave function. Putting $\widetilde{\widetilde{a}}^{a}$ (which has the property that $\left[\widetilde{S}^{a b}, \widetilde{\widetilde{a}}^{c}\right]=0$ ) equal strongly to zero is the same as replacing $\mathcal{S}^{a b}$ by $\widetilde{S}^{a b}$.

In both approaches to obtain spinors from vectors and antisymmetric tensors one starts by getting not only one but several copies, families, of Dirac fields. This is a fundamental feature insofar as these different families are connected by the generator parts not used: if, for instance, one uses $\widetilde{S}^{a b}$ as the Lorentz generator to obtain spinors, then the unused part $\widetilde{\widetilde{S}}^{a b}$ transforms the families (of the same Grassmann character) into each other.

It will be a major feature of the present article to create a dictionary relating the two formalisms so that one can enjoy the simplicity of one, which also is working on the other approach. We shall also generalize the Kähler operators for $d \neq 4$, comment on the discrete symmetries, which in the approach of one of us, show up clearly and use the $d-4$ dimensions to describe spins and charges [2-4].

In the following section we shall put forward the small part of the formalism of the work of one of us needed for the comparison with the Dirac-Kähler formalism. In Sec. III we shall then explain the (usual) Dirac-Kähler formalism as far as it is relevant. The comparison, which should now be rather obvious, is performed in Sec. IV. ${ }^{1}$

In Sec. $\mathrm{V}$ we shall analyze in the two approaches in parallel how the remarkable finding of the Dirac equation inside

\footnotetext{
${ }^{1}$ After submitting this paper to Phys. Rev. D, an interesting paper [5] appeared, in which the author compares the Dirac-Kähler approach to the symplectic geometry of phase spaces. Some of the author's conclusions are similar to ours. However, while Reuter extends the Dirac-Kähler approach to the space of anticommuting coordinates, we use the approach of one of us to generalize the Kähler approach.
} 
a purely tensorial-vectorial system of fields occurs. In Sec. VI we shall comment on the evenness of the $\gamma^{a}$ matrices, which have to transform Grassmann odd wave functions into Grassmann odd wave functions, not changing the Grassmann character of wave functions. In Sec. VII we shall comment on discrete symmetries, for either the Kähler approach, or the approach of one of us, also discussing the realization of the discrete symmetries pointed out clearly by Weinberg in his book [6] on pages 100-105. In Sec. VIII we want to investigate how unavoidable the appearance of families is to this type of approach. In Sec. IX we shall look at how the ideas of one of us of extra dimensions generalizes the Kähler approach. In Sec. X we discuss the Nielsen and Ninomija [7] no go theorem for spinors on a lattice and a possible way out. In Sec. XI we shall summarize, and deliver concluding remarks.

\section{DIRAC EQUATIONS IN GRASSMANN SPACE}

What we can call the Mankoč approach [2-4], and which is the work of one of us, is a rather ambitious model for going beyond the Standard model with, say, 10 (or more) extra dimensions, but what we need for the present connection with the Dirac-Kähler [1] formalism is only the way in which the spin part of the Dirac particle fields comes about. The total number of dimensions in the model is (most hopefully) $13+1$ bosonic degrees of freedom, i.e., normal dimensions, and the same number of fermionic ones.

Let us call the dimension of space-time $d$ and then the Dirac spinor degrees of freedom will come from the odd Grassmannian variables $\theta^{a}, a \in\{0,1,2,3,5, \ldots, d\}$.

In wanting to quantize or just to make Poisson brackets out of the $d \theta^{a}$ 's we have two choices, since we could either decide to make the different $\theta^{a}$ 's their own conjugate, so that one only has $d / 2$ degrees of freedom-this is the approach of Ravndal and DiVecchia [8] — or we could decide to consider the $\theta^{a}$ 's configuration space variables only. In the latter case-which is the Mankoč case-we have then from the $\theta^{a}$ 's different conjugate variables $p^{\theta a}$.

In this latter case we are entitled to write wave functions of the form

$$
\begin{aligned}
\psi\left(\left\{\theta^{a}\right\}\right)= & \sum_{i=0,1, \ldots, 3,5, \ldots, d} \sum_{\left\{a_{1}<a_{2}<\ldots<a_{i}\right\} \in\{0,1, \ldots, 3,5, \ldots, d\}} \\
& \times \alpha_{a_{1}, a_{2}, \ldots, a_{i}} \theta^{a_{1}} \theta^{a_{2}} \ldots \theta^{a_{i}}
\end{aligned}
$$

This is the only form a function of the odd Grassmannian variables $\theta^{a}$ can take. Thus the wave function space here has dimension $2^{d}$. Completely analogously to usual quantum mechanics we have the operator for the conjugate variable $\theta^{a}$ as

$$
p_{a}^{\theta}:=-i \frac{\vec{\partial}}{\partial \theta^{a}}:=-i \vec{\partial}_{a}
$$

The right arrow here just tells us, that the derivation has to be performed from the left-hand side. These operators then obey the odd Heisenberg algebra which, written by means of the generalized commutators

$$
\{A, B\}:=A B-(-1)^{n_{A B} B A,}
$$

where

$$
n_{A B}= \begin{cases}+1, & \text { if } A \text { and } B \text { have Grassmann odd character, } \\ 0 & \text { otherwise }\end{cases}
$$

takes the form

$$
\left\{p^{\theta a}, p^{\theta b}\right\}=0=\left\{\theta^{a}, \theta^{b}\right\}, \quad\left\{p^{\theta a}, \theta^{b}\right\}=-i \eta^{a b} .
$$

Here $\eta^{a b}$ is the flat metric $\eta=\operatorname{diag}\{1,-1,-1, \ldots\}$.

For later use we shall define the operators

$$
\widetilde{a}^{a}:=i\left(p^{\theta a}-i \theta^{a}\right), \quad \widetilde{\widetilde{a}}^{a}:=-\left(p^{\theta a}+i \theta^{a}\right),
$$

for which we can show that the $\widetilde{a}^{a}$ 's among themselves fulfill the Clifford algebra, as do also the $\widetilde{\widetilde{a}}^{a}$ 's, while they mutually anticommute:

$$
\left\{\widetilde{a}^{a}, \widetilde{a}^{b}\right\}=2 \eta^{a b}=\left\{\widetilde{\widetilde{a}}^{a}, \widetilde{\tilde{a}}^{b}\right\}, \quad\left\{\widetilde{a}^{a}, \widetilde{\tilde{a}}^{b}\right\}=0 .
$$

Note that the linear combinations (6) presuppose a metric tensor, since otherwise only $\theta^{a}$ and $p^{\theta}{ }_{a}$ but not $\theta_{a}$ and $p^{\theta a}$ are defined.

We could recognize formally

$$
\text { either } \widetilde{a}^{a} p_{a}|\psi\rangle=0 \text { or } \tilde{\widetilde{a}}^{a} p_{a}|\psi\rangle=0
$$

as the Dirac-like equation, because of the above generalized commutation relations. Applying either the operator $\widetilde{a}^{a} p_{a}$ or $\tilde{\widetilde{a}}^{a} p_{a}$ on the two equations [Eqs. (8)] we get the KleinGordon equation $p^{a} p_{a}|\psi\rangle=0$. Here of course we defined

$$
p_{a}=i \frac{\partial}{\partial x^{a}}
$$

However, it is rather obvious that Eqs. (8) are not Dirac equations in the sense of the wave function transforming as a spinor, with respect to the generators for the Lorentz transformations, if taken as usual

$$
\mathcal{S}^{a b}:=\theta^{a} p^{\theta b}-\theta^{b} p^{\theta a}
$$

However it is easily seen that we can write these generators as the sum

$$
\mathcal{S}^{a b}=\widetilde{S}^{a b}+\widetilde{\widetilde{S}}^{a b},
$$

where we have defined

$$
\widetilde{S}^{a b}:=-\frac{i}{4}\left[\widetilde{a}^{a}, \widetilde{a}^{b}\right], \quad \widetilde{S}^{a b}:=-\frac{i}{4}\left[\widetilde{\widetilde{a}}^{a}, \widetilde{\widetilde{a}}^{b}\right]
$$

with $[A, B]:=A B-B A$. One can now easily see that the solutions of the two equations (8) now transform as spinors with respect to either $\widetilde{S}^{a b}$ or $\widetilde{\widetilde{S}}^{a b}$.

It is of great importance for the "trick" of manipulating what we shall consider to be the Lorentz transformations, and thus to be able to make the "miraculous" shifts of 
Lorentz representations that is the somewhat remarkable characteristic of the Kähler type of shift in formulation interpretation, that-untilded, the single tilded and the double tilded $-\mathcal{S}^{a b}$ obey the $d$-dimensional Lorentz generator algebra $\left\{M^{a b}, M^{c d}\right\}=-i\left(M^{a d} \eta^{b c}+M^{b c} \eta^{a d}-M^{a c} \eta^{b d}\right.$ $\left.-M^{b d} \eta^{a c}\right)$, when inserted for $M^{a b}$.

Really the "cheat" consists of replacing the Lorentz generators by the $\widetilde{S}^{a b}$, say (we shall return to this later). This "cheat" means indeed that for this choice the operators $\widetilde{a}^{a}$ have to be put strongly to zero in the generators of the Lorentz transformations [Eqs. (10)-(12)] as well as in all the other operators, representing the physical quantities. We shall present the approach of one of us, in further detail in Sec. IV, pointing out the similarities between this approach and the Kähler one, and generalizing the Kähler approach.

\section{KÄHLER FORMULATION OF SPINORS}

The Kähler formulation [1] takes its starting point by considering $p$ forms in the $d$-dimensional space, $d=4$. Elegantly, the 1 forms say are defined as dual vectors to the (local) tangent spaces, and the higher $p$ forms can then be defined as antisymmetrized Cartesian (exterior) products of the one-form spaces, and the 0 forms are the scalars; but we can perhaps more concretely think about the $p$ forms as formal linear combinations of the differentials of the coordinates $d x^{a}$ : a general linear combination of forms is then written

$$
u=u_{0}+u_{1}+\cdots+u_{d},
$$

where the $p$ form is

$$
\begin{aligned}
u_{p} & =\frac{1}{p !} \sum_{i_{1}, i_{2}, \ldots, i_{p}} a_{i_{1}, i_{2}, \ldots, i_{p}} d x^{i_{1}} \wedge d x^{i_{2}} \wedge d x^{i_{3}} \wedge \ldots \wedge d x^{i_{p}} \\
& =\sum_{i_{1}<i_{2} \cdots<i_{p}} a_{i_{1}, i_{2}, \ldots, i_{p}} d x^{i_{1}} \wedge d x^{i_{2}} \bigwedge d x^{i_{3}} \wedge \ldots \wedge d x^{i_{p}} .
\end{aligned}
$$

Then one can define both the presumably most wellknown exterior algebra denoted by the exterior product $\wedge$ and the Clifford product $\vee$ among the forms. The wedge product $\wedge$ has the property of making the product of a $p$ form and a $q$ form be a $(p+q)$ form, if a $p$ form and a $q$ form have no common differentials. The Clifford product $d x^{a} \vee$ on a $p$ form is either a $p+1$ form, if a $p$ form does not include a one form $d x^{a}$, or a $p-1$ form, if a one form $d x^{a}$ is included in a $p$ form.

Actually Kähler found how the Dirac equation could be written as an equation [1] [Eq. (26.6) in Kähler's paper]

$$
-i \delta u=(m+e \cdot \omega) \bigvee u,
$$

where the symbol $u$ stands for a linear combination of $p$ forms [Eq. (13)] and $p \in\{0,1, \ldots, 3,5, \ldots, d\}$ with $d$ being the dimension of space-time, namely, $d=4$ for Kähler's case. Further in the notation of Kähler the symbol ${ }^{2} \delta$ denotes inner differentiation, which means the analogue of the exterior differential $d$ but with the use of the Clifford product $V$ instead of the exterior product $\wedge$

$$
\delta u=\sum_{i=1}^{3} d x^{i} \bigvee \frac{\partial u}{\partial x^{i}}-d t \bigvee \frac{\partial u}{\partial t}=d u+\sum_{i=1}^{3} e_{i} \frac{\partial u}{\partial x^{i}}-e_{0} \frac{\partial u}{\partial t} .
$$

$e \omega$ determines the coupling of the charge with the electromagnetic field $\omega=A_{a} d x^{a}, a \in\{0,1,2,3\}$ and $m$ means the electron mass; the symbol $e_{i}$ transforms a $p$ form into ( $p$ -1 ) form, if the $p$ form includes $d x^{i}$, otherwise it gives zero.

For a free massless particle living in a $d$-dimensional space-time- this is what interests us in this paper since the mass term brings no new feature to the theory-Eq. (15) can be rewritten in the form

$$
d x^{a} \vee p_{a} u=0, \quad a=0,1,2,3,5, \ldots, d,
$$

where $u$ again stands for a linear combination of $p$ forms $(p=0,1,2,3,5, \ldots, d)$, that is to say that the wave function describing the state of the spin-one-half particle is packed into the exterior algebra function $u$. More about the Kähler approach will come in Sec. IV, giving the correspondence between that and the one with the Grassmann $\theta^{a}$ s, where we shall also give some generalizations.

\section{PARALLELISM BETWEEN THE TWO APPROACHES}

We demonstrate the parallelism between the Kähler approach [1] and that of one of us [2-4] in steps, paying attention to the Becher-Joos [9] paper as well. First we shall treat the spin- $\frac{1}{2}$ fields only, as Kähler did. We shall use the simple and transparent definition of the exterior and interior products in Grassmann space to generalize the Kähler approach to two kinds of $\delta$ [Eq. (16)] operators on the space of $p$ forms and then accordingly to three kinds of the generators of the Lorentz transformations, two of the spinorial and one of the vectorial character, the first kind transforming spinor $-\frac{1}{2}$ fields, the second one transforming the vector fields. We comment on the Hodge star product for both approaches, define the scalar product of vectors in the vector space of either $p$ forms or of polynomials of $\theta^{a}$ 's and comment on four replications of the Weyl bispinor. We also discuss briefly the vector representations in both approaches.

\section{A. Dirac-Kähler equation and Dirac equation in Grassmann space for massless particles}

We present here, side by side, the operators in the space of differential forms and in the space of polynomials of $\theta^{a}$ 's. We present the exterior product

\footnotetext{
${ }^{2}$ The notation in the Becher and Joos [9] paper is slightly different from the Kähler notation. The Becher and Joos paper uses $d$ $=d x^{a}\left(\partial / \partial x^{a}\right)$, as Kähler does in his paper, but $\delta$ of Kähler is in the Becher and Joos paper replaced by $d-\delta$, which means that in their paper $\delta=-e^{a}\left(\delta / \delta x^{a}\right)$.
} 


$$
d x^{a} \wedge d x^{b} \wedge \cdots, \quad \theta^{a} \theta^{b} \cdots,
$$

the operator of "differentiation",

$$
-i e^{a}, \quad p^{\theta a}=-i \vec{\partial}^{a}=-i \frac{\vec{\partial}}{\partial \theta_{a}}
$$

and the two superpositions of the above operators

$$
\begin{aligned}
& d x^{a} \widetilde{\vee}:=d x^{a} \wedge+e^{a}, \quad \widetilde{a}^{a}:=i\left(p^{\theta a}-i \theta^{a}\right), \\
& d x^{a} \widetilde{\nabla}:=-i\left(d x^{a} \wedge-e^{a}\right), \quad \widetilde{a}^{a}:=-\left(p^{\theta a}+i \theta^{a}\right) .
\end{aligned}
$$

Here $\widetilde{V}$ stays instead of $\vee$ of Eq. (15), used by Kähler. Introducing the notation with $(\sim)$ and $(\widetilde{\sim})$ we not only point out the similarities between the two approaches but also the two possibilities for the Clifford product-only one of them used by Kähler. Both $\tilde{\nabla}$ and $\widetilde{\widetilde{V}}$ are Clifford products on $p$ forms, while $\widetilde{a}^{a}$ and $\widetilde{a}^{a}$ are the corresponding linear operators operating on the space of polynomials of $\theta^{a}$ 's. One easily finds the commutation relations, if for both approaches the generalized form of commutators, presented in Eq. (4), are understood

$$
\begin{aligned}
& \left\{d x^{a} \tilde{\vee}, d x^{b} \tilde{\vee}\right\}=2 \eta^{a b}, \quad\left\{\tilde{a}^{a}, \tilde{a}^{b}\right\}=2 \eta^{a b} \\
& \left\{d x^{a} \approx \vec{\nabla}, d x^{b} \widetilde{\nabla}\right\}=2 \eta^{a b}, \quad\left\{\widetilde{\widetilde{a}}^{a}, \widetilde{\widetilde{a}}^{b}\right\}=2 \eta^{a b} .
\end{aligned}
$$

Here $\eta^{a b}$ is the metric of space-time.

The vacuum state |\rangle is defined as

$$
\begin{aligned}
& d x^{a} \tilde{\vee}|\rangle=d x^{a} \wedge, \quad \tilde{a}^{a}|\rangle=\theta^{a}, \\
& d x^{a} \approx \vec{\vee}|\rangle=-i d x^{a} \wedge, \quad \widetilde{a}^{a}|\rangle=-i \theta^{a} .
\end{aligned}
$$

Now we can define the Dirac-like equations for both approaches

$$
\begin{aligned}
& d x^{a} \widetilde{\vee} p_{a} u=0, \quad \tilde{a}^{a} p_{a} \psi\left(\left\{\theta^{a}\right\}\right)=0, \\
& d x^{a} \approx \tilde{\nabla}_{a} u=0, \quad \tilde{\widetilde{a}}^{a} p_{a} \psi\left(\left\{\theta^{a}\right\}\right)=0 .
\end{aligned}
$$

Since $\left\{e^{a}, d x^{b} \wedge\right\}=\eta^{a b}$ and $\left\{e^{a}, e^{b}\right\}=0=\left\{d x^{a} \wedge, d x^{b} \wedge\right\}$ $=0$, while $\left\{i p^{\theta a}, \theta^{b}\right\}=\eta^{a b}$ and $\left\{i p^{\theta a}, i p^{\theta b}\right\}=0=\left\{\theta^{a}, \theta^{b}\right\}$, it is obvious that in the $p$ form formalism $e^{a}$ plays the role of the derivative with respect to a differential one form, similarly as $i p^{\theta a}$ does with respect to a Grassmann coordinate.

Taking into account the above definitions, one easily finds that

$$
\begin{aligned}
& d x^{a} \tilde{\vee} p_{a} d x^{b} \tilde{\vee} p_{b} u=p^{a} p_{a} u=0, \\
& \widetilde{a}^{a} p_{a} \widetilde{a}^{b} p_{b} \psi\left(\left\{\theta^{b}\right\}\right)=p^{a} p_{a} \psi\left(\left\{\theta^{b}\right\}\right)=0 . \\
& d x^{a} \approx \tilde{\vee}_{a} d x^{b} \approx \tilde{\vee}_{b} u=p^{a} p_{a} u=0, \\
& \widetilde{\widetilde{a}}^{a} p_{a} \widetilde{\widetilde{a}}^{b} p_{b} \psi\left(\left\{\theta^{b}\right\}\right)=p^{a} p_{a} \psi\left(\left\{\theta^{b}\right\}\right)=0 .
\end{aligned}
$$

Both vectors, the $u$, which are the superpositions of differential $p$ forms and the $\psi\left(\theta^{a}\right)$, which are polynomials in $\theta^{a}$ 's, are defined in a similar way [Eqs. (1),(14)], as we shall point out in the following subsection. We see that either

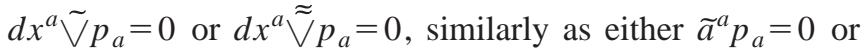
$\widetilde{\widetilde{a}}^{a} p_{a}=0$ can represent the Dirac-like equation.

\section{B. Vector space of two approaches}

The superpositions of $p$ forms on which the Dirac-Kähler equation is defined and the superpositions of polynomials in Grassmann space, on which the Dirac-like equations are defined, are

$$
\begin{aligned}
& u=\sum_{i=0,1,2,3,5, \ldots, d} \sum_{a_{1}<a_{2}<\ldots<a_{i} \in\{0,1,2,3,5, \ldots, d\}} \\
& \times a_{a_{1}, a_{2}, \ldots, a_{i}} d x^{a_{1}} \wedge d x^{a_{2}} \wedge d x^{a_{3}} \wedge \ldots \wedge d x^{a_{i}}, \\
& \psi\left(\theta^{a}\right)=\sum_{i=0, d} \sum_{a_{1}<a_{2}<\ldots<a_{i} \in\{0,1,2,3,5, \ldots, d\}} \\
& \times \alpha_{a_{1}, a_{2}, \ldots, a_{i}} \theta^{a_{1}} \theta^{a_{2}} \ldots \theta^{a_{i}} .
\end{aligned}
$$

The coefficients $\alpha_{a_{1}, a_{2}, \ldots, a_{i}}$ depend on coordinates $x^{a}$ in both cases and are antisymmetric tensors of the rank $i$ with respect to indices $a_{k} \in\left\{a_{1}, \ldots, a_{i}\right\}$. The vector space is for $d=4$, used by Kähler, in both cases 16 dimensional.

\section{Dirac $\gamma^{a}$-like operators}

Both $d x^{a} \widetilde{\nabla}$ and $d x^{a} \approx \widetilde{\nabla}$ define the algebra of the $\gamma^{a}$ matrices and so do both $\widetilde{a}^{a}$ and $\widetilde{a}^{a}$. One would thus be tempted to identify

$$
\gamma_{\text {naive }}^{a}:=d x^{a} \widetilde{\vee} \text {, or } \gamma_{\text {naive }}^{a}:=\widetilde{a}^{a}
$$

But there is a large freedom in defining what to identify with the gamma matrices, because except when using $\gamma^{0}$ as a parity operation you have an even number of gamma matrices occurring in the physical applications. ${ }^{3}$ Then you may multiply all the gamma matrices by some factor provided it

\footnotetext{
${ }^{3}$ There are currents $\bar{\psi} \gamma^{a} \psi$ which enter into calculations of physical quantities. Currents have an even number of $\gamma^{a}$ matrices: $\psi^{+} \gamma^{0} \gamma^{a} \psi$. Also the Lagrange function contains, in addition to the mass term, in which $\gamma^{0}$ appears $\left(m \bar{\psi} \psi=m \psi^{+} \gamma^{0} \psi\right)$, the even number of $\gamma^{a}$ matrices: $\bar{\psi} \gamma^{a} p_{a}$.
} 
does not disturb their algebra or their even products. We shall comment on this point in Sec. VI.

\section{Problem of statistics of gamma matrices}

This freedom might be used to solve what seems to be a problem. Having an odd Grassmann character, neither $\widetilde{a}^{a}$ nor $\widetilde{a}^{a}$ should be recognized as the Dirac $\gamma^{a}$ operators since, when operating on polynomials of $\theta^{a}$, they would change polynomials of an odd Grassmann character to polynomials of an even Grassmann character. One would, however, expect-since Grassmann odd fields second-quantize to fermions, while Grassmann even fields second-quantize to bosons-that the $\gamma^{a}$ operators do not change the Grassmann character of wave functions. One can notice that, similarly to the Grassmann case, also the two types of the Clifford products defined on $p$ forms, change the oddness or the evenness of the $p$ forms: an even $p$ form, $p=2 n$, is changed by either $d x^{a} \widetilde{V}$ or $d x^{a} \widetilde{\widetilde{V}}$ to an odd $q$ form, with either $q=p+1$, if $d x^{a}$ is not included in a $p$ form, or $q=p-1$, if $d x^{a}$ is included in a $p$ form, while an odd $p$ form, $p=2 n+1$, is changed to an even $p+1$ form or $p-1$ form.

\section{First solution to gamma-matrix statistics problem}

We shall later therefore propose that accordingly

$$
\text { either } \tilde{\gamma}^{a}:=i d x^{0} \widetilde{\nabla} d x^{a} \tilde{\vee} \text { or } \tilde{\gamma}^{a}=i \widetilde{a}^{0} \widetilde{a}^{a}
$$

are recognized as the Dirac $\gamma^{a}$ operators operating on the space of $p$ forms or polynomials of $\theta^{a}$ 's, respectively, since they both have an even Grassmann character and they both fulfill the Clifford algebra

$$
\left\{\tilde{\gamma}^{a}, \tilde{\gamma}^{b}\right\}=2 \eta^{a b}
$$

Of course, the roles of $(\sim)$ and $(\widetilde{\sim})$ can be exchanged in either the Kähler case or the case of polynomials in Grassmann space.

Whether we define the gamma matrices by Eq. (27) or (26) only makes a difference for an odd product of gamma matrices, but for applications (see the last footnote) such as construction of currents $\bar{\psi} \gamma^{a} \psi$ or for the Lorentz generators on the spinors $-i \frac{1}{4}\left[\gamma^{a}, \gamma^{b}\right]$ only products of even numbers of gamma matrices occur, except for the parity representation on the Dirac fields, where the $\gamma^{0}$ matrix is used alone. This $\gamma^{0}$ matrix has to simulate the parity reflection, which is either

$$
\vec{d} x \rightarrow-\vec{d} x \text { or } \vec{\theta} \rightarrow-\vec{\theta} .
$$

The "ugly" gamma-matrix identifications (27) indeed perform this operation. And as long as the physical applications are the ones just mentioned-and that should be sufficientthe choice (27) is satisfactory: living in the Grassmann odd part of the Hilbert space, we do not move into the Grassmann even part of it. The canonical quantization of Grassmann odd fields, that is the procedure with the Hamiltonian and the Poisson brackets, then automatically assures the anticommuting relations between the operators of the fermionic fields.

\section{Solution by redefinition of oddness}

The simplest solution to the problem with the evenness and oddness is to use the "naive" gamma matrix identifications (26) and simply ignore that the even-odd-ness does not match. This is what Kähler did, we can say, insofar as he did not really identify the even-odd-ness of the $p$ forms with the statistics of Dirac fields. If, along the lines of the Becher and Joos paper [9], one makes a second-quantized theory based on the Kähler trick, one does not proceed by insisting on taking $p$ forms to be fermionic only when $p$ is odd. Becher and Joos take all the forms as fermion fields and then assume anticommuting relations for operators of fields. This simplest solution can thus be claimed to be the one applied by Kähler and used by Becher and Joos: they simply do not dream about postulating in advance that the $p$ forms should necessarily be taken to be boson or fermion fields depending on whether $p$ is even or odd. It is only when one as one of us in her model has the requirement of canonical quantization saying that the $\theta^{a}$ 's should be Grassmann odd objects, which indeed they are, that the problem occurs.

\section{Generators of Lorentz transformations}

Again we present the generators of the Lorentz transformations of spinors for both approaches

$$
M^{a b}=L^{a b}+\mathcal{S}^{a b}, L^{a b}=x^{a} p^{b}-x^{b} p^{a},
$$

differing among themselves in the definition of $\mathcal{S}^{a b}$ only, which define the generators of the Lorentz transformations in the internal space, that is in the space of $p$ forms or polynomials of $\theta^{a}$ s, respectively. While Kähler suggested the definition

$$
\begin{aligned}
\mathcal{S}^{a b} & =d x^{a} \wedge d x^{b}, \\
\mathcal{S}^{a b} u & =\frac{1}{2}\left[\left(d x^{a} \wedge d x^{b}\right) \bigvee u-u \vee\left(d x^{a} \wedge d x^{b}\right)\right],
\end{aligned}
$$

in the Grassmann case [2] the operator $\mathcal{S}^{a b}$ is one of the two generators defined above [Eq. (12)], that is,

$$
\begin{aligned}
\text { either } \mathcal{S}^{a b} & =\widetilde{S}^{a b}=-\frac{i}{4}\left[\widetilde{a}^{a}, \widetilde{a}^{b}\right]=-\frac{i}{4}\left[\tilde{\gamma}^{a}, \tilde{\gamma}^{b}\right] \\
\text { or } \mathcal{S}^{a b} & =\widetilde{\widetilde{S}}^{a b}=-\frac{i}{4}\left[\widetilde{a}^{a}, \widetilde{a}^{b}\right] .
\end{aligned}
$$

One further finds 


$$
\begin{array}{r}
{\left[\widetilde{S}^{a b}, \tilde{a}^{c}\right]=i\left(\eta^{a c} \tilde{a}^{b}-\eta^{b c} \widetilde{a}^{a}\right),} \\
{\left[\widetilde{\widetilde{S}}^{a b}, \widetilde{\widetilde{a}}^{c}\right]=i\left(\eta^{a c} \widetilde{\widetilde{a}}^{b}-\eta^{b c} \widetilde{\widetilde{a}}^{a}\right),} \\
\text { while }\left[\widetilde{S}^{a b}, \widetilde{\widetilde{a}}^{c}\right]=0=\left[\widetilde{\widetilde{S}}^{a b}, \widetilde{a}^{c}\right] .
\end{array}
$$

In the Kähler case one can also define two kinds of Lorentz generators, which operate on the internal space of $p$ forms, according to two kinds of Clifford products, presented above. Following the definitions in the approach of one of us [2], one can write the $\mathcal{S}^{a b}$ for the Kähler case: either

$$
\begin{aligned}
\widetilde{S}^{a b} & =-\frac{i}{4}\left[d x^{a} \wedge+e^{a}, d x^{b} \wedge+e^{b}\right]=-\frac{i}{4}\left[\tilde{\gamma}^{a}, \tilde{\gamma}^{b}\right] \\
\text { or } \widetilde{\widetilde{S}}^{a b} & =\frac{i}{4}\left[d x^{a} \wedge-e^{a}, d x^{b} \wedge-e^{b}\right] .
\end{aligned}
$$

Not only in this case, are the similarities between the two approaches more transparent, but also the definition of the generators of the Lorentz transformations in the space of $p$ forms is very much simplified.

One further finds for the spinorial case

$$
\left[M^{a b}, \tilde{\gamma}^{a} p_{a}\right]=0 \text { for } M^{a b}=L^{a b}+\widetilde{S}^{a b},
$$

which demonstrates that the total angular momentum for a free massless particle is conserved. The above equation is true for both approaches and the generators of the Lorentz transformations $M^{a b}$ fulfill the Lorentz algebra in both cases.

In addition, the operators of the Lorentz transformations with the vectorial character can also be defined for both approaches in an equivalent way, that is as a sum of the two operators of the spinorial character

$$
\begin{aligned}
& \mathcal{S}^{a b}=\widetilde{S}^{a b}+\widetilde{\widetilde{S}}^{a b}=-i\left(d x^{a} \wedge e^{b}-d x^{b} \wedge e^{a}\right), \\
& \mathcal{S}^{a b}=\widetilde{S}^{a b}+\widetilde{\widetilde{S}}^{a b}=\theta^{a} p^{\theta b}-\theta^{b} p^{\theta a},
\end{aligned}
$$

which again fulfills the Lorentz algebra. The operator $\mathcal{S}^{a b}$ $=-i\left(d x^{a} \wedge e^{b}-d x^{b} \wedge e^{a}\right)$, if applied on differential $p$ forms, transforms vectors into vectors; correspondingly, $\mathcal{S}^{a b}$ $=\theta^{a} p^{\theta b}-\theta^{b} p^{\theta a}$, if applied to polynomials of $\theta^{a}$ transforms vectors into vectors [2].

Elements of the Lorentz group can be written for both approaches, for either spinorial or vectorial kinds of generators as

$$
U=e^{-(i / 2) \omega_{a b} M^{a b}}
$$

where $\omega_{a b}$ are parameters of the group. If $M^{a b}$ are equal to either $L^{a b}+\widetilde{S}^{a b}$ or $L^{a b}+\widetilde{\widetilde{S}}^{a b}$, the period of transformation is $4 \pi$ either in the space of differential forms or in the Grassmann space, demonstrating the spinorial character of the operator. If $M^{a b}$ is the sum of $L^{a b}$ and $\widetilde{S}^{a b}+\widetilde{\widetilde{S}}^{a b}$, the period of transformation is $2 \pi$, manifesting the vectorial character of the operator.

\section{E. Hodge star product}

In the way in which we have defined the operators in the space of $p$ forms, the definition of the "Hodge star'" operator, defined by Kähler working in the space of $p$ forms and the space of $\theta^{a}$ polynomials, will be, respectively,

$$
\widetilde{\Gamma}=i \prod_{a=0,1,2,3,5, \ldots, d} \tilde{\gamma}^{a}
$$

with $\tilde{\gamma}^{a}$ equal to either $i d x^{0} \approx \widetilde{\nabla} d x^{a} \widetilde{\nabla}$ in the Kähler case, or to $i \widetilde{a}^{0} \widetilde{a}^{a}$ in the approach of one of us [2]. For an even $d$ the factors with double tildes can in both cases be omitted $(\widetilde{\Gamma}$ $=$ either $i \Pi_{a} \widetilde{a}^{a}$ or $i \Pi_{a} d x^{a} \widetilde{\vee}$; again we could distinguish the operators $\widetilde{\Gamma}$ and $\widetilde{\Gamma}$ in both cases, according to the elements, which define the Casimir). It follows that

$$
\frac{1}{2}(1 \pm \widetilde{\Gamma})
$$

are the two operators, which when being applied on wave functions defined either on $p$ forms or on polynomials in Grassmann space, project out the left- or right-handed component, respectively.

One easily recognizes that when being applied on a vacuum state |\rangle , the operator $\widetilde{\Gamma}$ behaves as a "Hodge star"' product, since one finds, for $d$ even,

$$
-i \widetilde{\Gamma}|\rangle=d x^{0} \wedge d x^{1} \wedge \cdots \wedge d x^{d}, \quad-i \widetilde{\Gamma}|\rangle=\theta^{0} \theta^{1} \cdots \theta^{d}
$$

\section{F. Scalar product}

In Mankoč's approach [2], the scalar product between the two functions $\psi^{(1)}\left(\left\{\theta^{a}\right\}\right)$ and $\psi^{(2)}\left(\left\{\theta^{a}\right\}\right)$ is defined as follows:

$$
\left\langle\psi^{(1)} \mid \psi^{(2)}\right\rangle=\int d^{d} \theta\left[\omega \psi^{(1)}\left(\left\{\theta^{a}\right\}\right)\right]^{*} \psi^{(2)}\left(\left\{\theta^{a}\right\}\right) .
$$

Here $\omega$ is the weight function

$$
\omega=\prod_{i=0,1,2,3,5, \ldots, d}\left(\theta^{i}+\frac{\vec{\partial}}{\partial \theta^{i}}\right)
$$

which operates on the first function $\psi^{(1)}$ only, while

$$
\int d \theta^{a}=0, \quad \int d \theta^{a} \theta^{a}=1, \quad a=0,1,2,3,5, \ldots, d,
$$

no summation over the repeated index is meant and

$$
\begin{aligned}
\int d^{d} \theta \theta^{0} \theta^{1} \theta^{2} \theta^{3} \theta^{5} \cdots \theta^{d} & =1, \\
d^{d} \theta & =d \theta^{d} \cdots d \theta^{5} d \theta^{3} d \theta^{2} d \theta^{1} d \theta^{0} .
\end{aligned}
$$

Since $\theta^{a *}=\theta^{a}$, an asterisk means the complex conjugation and a plus means the Hermitian conjugation, then with re- 
spect to the scalar product defined above the operator $\theta^{a+}$ $=\eta^{a a} \vec{\partial}^{a}, \vec{\partial}^{a+}=\theta^{a} \eta^{a a}$, while $\widetilde{a}^{a+}=\eta^{a a} \widetilde{a}^{a}$ and $\widetilde{\widetilde{a}}^{a+}=\eta^{a a} \widetilde{\widetilde{a}}^{a}$. Again no summation over the repeated index is performed. Accordingly the operators of the Lorentz transformations of spinorial character are self-adjoint (if $a \neq 0$ and $b \neq 0$ ) or anti-self-adjoint (if $a=0$ or $b=0$ ).

According to Eqs. (41), (25) the scalar product of two functions $\psi^{(1)}\left(\theta^{a}\right)$ and $\psi^{(2)}\left(\theta^{a}\right)$ can be written as follows:

$$
\left\langle\psi^{(1)} \mid \psi^{(2)}\right\rangle=\sum_{0, d} \sum_{\alpha_{1}<\alpha_{2}<\ldots<\alpha_{i}} \alpha_{\alpha_{1}, \alpha_{2}, \ldots, \alpha_{i}}^{(1) *} \alpha_{\alpha_{1}, \alpha_{2}, \ldots, \alpha_{i}}^{(2)}
$$

in complete analogy with the usual definition of scalar products in ordinary space. Kähler [1] defined in Eq. (15.11) and on page 519 the scalar product of two superpositions of $p$ forms $u^{(1)}$ and $u^{(2)}$ as follows:

$$
\left\langle u^{(1)} \mid u^{(2)}\right\rangle=\sum_{0, d} \sum_{\alpha_{1}<\alpha_{2}<\ldots<\alpha_{i}} \alpha_{\alpha_{1}, \alpha_{2}, \ldots, \alpha_{i}}^{(1)} \alpha_{\alpha_{1}, \alpha_{2}, \ldots, \alpha_{i}}^{(2)}
$$

which (for real coefficients $\alpha_{\alpha_{1}, \alpha_{2}, \ldots, \alpha_{i}}^{(k)}, k=1,2$ ) agrees with Eq. (45).

\section{G. Four families of solutions in the Kähler or Grassmann space approach (of one of us)}

We shall limit ourselves to $d=4$ and to the spinorial case (as indeed Kähler did). The representations for higher $d$, analyzed with respect to the groups $\mathrm{SO}(1,3) \times \mathrm{SU}(3) \times \mathrm{SU}(2)$ $\times \mathrm{U}(1)$, and some other groups in Grassmann space, are for Grassmann even and Grassmann odd parts of the space, which belongs to the groups not including $\mathrm{SO}(1,3)$ presented in Ref. [3] and Ref. [4], respectively.

In the case of $d=4$ one may arrange the space of $2^{d}$ vectors into four times two Weyl spinors, one left-handed one $\left(\left\langle\widetilde{\Gamma}^{(4)}\right\rangle=-1\right)$ and one right $\left(\left\langle\widetilde{\Gamma}^{(4)}\right\rangle=1\right)$. We are presenting these vectors, which are at the same time the eigenvectors of $\widetilde{S}^{12}$ and $\widetilde{S}^{03}$, as polynomials of $\theta^{m}$ s, $m$ $\in(0,1,2,3)$. The two Weyl vectors are connected by the operation of $\tilde{\gamma}^{m}$ operators [Eq. (27)]. Taking into account that $\widetilde{a}^{a}|\rangle=\theta^{a}$, where |\rangle is the vacuum state [Eq. (22)], we present Table I.

Similarly also the Kähler spinors can be arranged into four copies. We find them by only replacing $\widetilde{a}^{a}$ by $d x^{a} \widetilde{V}$ in Table I. We shall discuss this point also in the next section.

\section{H. Vector representations of group $\mathrm{SO}(1,3)$}

Analyzing the irreducible representations of the group $\mathrm{SO}(1,3)$ in analogy with the spinor case but taking into account the generator of the Lorentz transformations of the vector type [Eqs. (10), (36)] one finds [2-4] for $d=4$, two scalars (a scalar and a pseudoscalar), two three-vectors [in the $\mathrm{SU}(2) \times \mathrm{SU}(2)$ representation of $\mathrm{SO}(1,3)$ usually denoted by $(1,0)$ and $(0,1)$ representations, respectively, with $\left\langle\Gamma^{(4)}\right\rangle$ equal to \pm 1$]$ and two four vectors [in the $\mathrm{SU}(2)$
TABLE I. Irreducible representations of the two subgroups $\mathrm{SU}(2) \times \mathrm{SU}(2)$ (i.e., the complex version of) the group $\mathrm{SO}(1,3)$ as defined by the generators of the spinorial character $\widetilde{S}^{12}, \widetilde{S}^{03}$ and the operator of handedness $\widetilde{\Gamma}^{(4)}$. The four copies of the Weyl bispinors have either an odd or an even Grassmann character. The generators $\widetilde{\widetilde{S}}^{m n}, m, n \in(0,1,2,3)$, transform the two copies of the same Grass-

\begin{tabular}{|c|c|c|c|c|c|}
\hline$a$ & $i$ & ${ }^{a} \widetilde{\psi}_{i}(\{\theta\})$ & $\widetilde{S}^{12}$ & $\widetilde{S}^{03}$ & $\widetilde{\Gamma}^{(4)}$ \\
\hline 1 & 1 & $\frac{1}{2}\left(\widetilde{a}^{1}-i \widetilde{a}^{2}\right)\left(\widetilde{a}^{0}-\widetilde{a}^{3}\right)$ & $\frac{1}{2}$ & $-\frac{i}{2}$ & -1 \\
\hline 1 & 2 & $-\frac{1}{2}\left(1+i \widetilde{a}^{1} \widetilde{a}^{2}\right)\left(1-\widetilde{a}^{0} \widetilde{a}^{3}\right)$ & $-\frac{1}{2}$ & $\frac{i}{2}$ & -1 \\
\hline 2 & 1 & $\frac{1}{2}\left(\widetilde{a}^{1}-i \widetilde{a}^{2}\right)\left(\widetilde{a}^{0}+\widetilde{a}^{3}\right)$ & $\frac{1}{2}$ & $\frac{i}{2}$ & 1 \\
\hline 2 & 2 & $-\frac{1}{2}\left(1+i \widetilde{a}^{1} \widetilde{a}^{2}\right)\left(1+\widetilde{a}^{0} \widetilde{a}^{3}\right)$ & $-\frac{1}{2}$ & $-\frac{i}{2}$ & 1 \\
\hline 3 & 1 & $\frac{1}{2}\left(\widetilde{a}^{1}-i \widetilde{a}^{2}\right)\left(1-\widetilde{a}^{0} \widetilde{a}^{3}\right)$ & $\frac{1}{2}$ & $\frac{i}{2}$ & 1 \\
\hline 3 & 2 & $-\frac{1}{2}\left(1+i \widetilde{a}^{1} \widetilde{a}^{2}\right)\left(\widetilde{a}^{0}-\widetilde{a}^{3}\right)$ & $-\frac{1}{2}$ & $-\frac{i}{2}$ & 1 \\
\hline 4 & 1 & $\frac{1}{2}\left(\widetilde{a}^{1}-i \widetilde{a}^{2}\right)\left(1+\widetilde{a}^{0} \widetilde{a}^{3}\right)$ & $\frac{1}{2}$ & $-\frac{i}{2}$ & -1 \\
\hline 4 & 2 & $-\frac{1}{2}\left(1+i \widetilde{a}^{1} \widetilde{a}^{2}\right)\left(\widetilde{a}^{0}+\widetilde{a}^{3}\right)$ & $-\frac{1}{2}$ & $\frac{i}{2}$ & -1 \\
\hline 5 & 1 & $\frac{1}{2}\left(1-i \widetilde{a}^{1} \widetilde{a}^{2}\right)\left(\widetilde{a}^{0}+\widetilde{a}^{3}\right)$ & $\frac{1}{2}$ & $\frac{i}{2}$ & 1 \\
\hline 5 & 2 & $-\frac{1}{2}\left(\widetilde{a}^{1}+i \widetilde{a}^{2}\right)\left(1+\widetilde{a}^{0} \widetilde{a}^{3}\right)$ & $-\frac{1}{2}$ & $-\frac{i}{2}$ & 1 \\
\hline 6 & 1 & $\frac{1}{2}\left(1-i \widetilde{a}^{1} \widetilde{a}^{2}\right)\left(\widetilde{a}^{0}-\widetilde{a}^{3}\right)$ & $\frac{1}{2}$ & $-\frac{i}{2}$ & -1 \\
\hline 6 & 2 & $-\frac{1}{2}\left(\widetilde{a}^{1}+i \widetilde{a}^{2}\right)\left(1-\widetilde{a}^{0} \widetilde{a}^{3}\right)$ & $-\frac{1}{2}$ & $\frac{i}{2}$ & -1 \\
\hline 7 & 1 & $\frac{1}{2}\left(1-i \widetilde{a}^{1} \widetilde{a}^{2}\right)\left(1+\widetilde{a}^{0} \widetilde{a}^{3}\right)$ & $\frac{1}{2}$ & $-\frac{i}{2}$ & -1 \\
\hline 7 & 2 & $-\frac{1}{2}\left(\widetilde{a}^{1}+i \widetilde{a}^{2}\right)\left(\widetilde{a}^{0}+\widetilde{a}^{3}\right)$ & $-\frac{1}{2}$ & $\frac{i}{2}$ & -1 \\
\hline 8 & 1 & $\frac{1}{2}\left(1-i \widetilde{a}^{1} \widetilde{a}^{2}\right)\left(1-\widetilde{a}^{0} \widetilde{a}^{3}\right)$ & $\frac{1}{2}$ & $\frac{i}{2}$ & 1 \\
\hline 8 & 2 & $-\frac{1}{2}\left(\widetilde{a}^{1}+i \widetilde{a}^{2}\right)\left(\widetilde{a}^{0}-\widetilde{a}^{3}\right)$ & $-\frac{1}{2}$ & $-\frac{i}{2}$ & 1 \\
\hline
\end{tabular}
mann character one into another.

$\times \mathrm{SU}(2)$ representation of $\mathrm{SO}(1,3)$ both denoted by $\left(\frac{1}{2}, \frac{1}{2}\right)$ and differing among themselves in the Grassmann character], all of which are eigenvectors of $\mathcal{S}^{(4) 2}=\frac{1}{2} \mathcal{S}^{a b} \mathcal{S}_{a b}, \Gamma^{(4)}$ $=i\left[(-2 i)^{2} / 4 !\right] \epsilon_{a b c d} \mathcal{S}^{a b} \mathcal{S}^{c d}, \mathcal{S}^{12}$, and $\mathcal{S}^{03}$. Using Eq. (36) and analyzing the vector space of $p$ forms in a way analogous to the space of the Grassmann polynomials, one finds the same kind of representations also in the Kähler case. Both in the spinor case and in the vector case one has $2^{4}$-dimensional vector space. 


\section{APPEARANCE OF SPINORS}

One may wonder about how it is at all possible that the Dirac equation-usually which is an equation for a spinor field-appears out of models with only scalar, vector, and tensor objects. Immediately one would say that it is of course purely impossible to construct spinors such as Dirac fields out of integer spin objects such as the differential one forms and their external products, or the $\theta^{a}$ 's and their products $\theta^{a} \theta^{b} \cdots \theta^{c}$.

Let us also say immediately that it can only be done by "cheating." This cheating really consists of replacing the Lorentz transformation concept (including rotation concept) by exchanging the Lorentz generators $\mathcal{S}^{a b}$ and $\widetilde{S}^{a b}$ say (or the $\widetilde{\widetilde{S}}^{a b}$ if we choose them instead), see Eqs. (32), (34). This indeed means that one of the two kinds of operators fulfilling the Clifford algebra and anticommuting with the other kind-a choice of $d x^{a} \approx \widetilde{\nabla}$ was made in the Kähler case and $\widetilde{\widetilde{a}}^{a}$ in the approach of one of us-are put to zero in the operators of Lorentz transformations as well as in all the operators representing the physical quantities. The use of $d x^{0} \approx \widetilde{\nabla}$ or $\widetilde{a}^{0}$ in the operator $\tilde{\gamma}^{0}$ is the exception, used only to simulate the Grassmann even parity operation $\vec{d} x^{a} \rightarrow-\vec{d} x^{a}$ and $\vec{\theta} \rightarrow-\vec{\theta}$, respectively. The assumption which we call "cheating" was made in the Kähler approach [1] and in its lattice version [9], as well as in the approach of one of us [2].

In Ref. [2] the $\widetilde{a}^{a}$ 's are argued away on the grounds, that with a certain single particle action

$$
I=\int d \tau d \xi L(x, \theta, \tau, \xi)
$$

(with $x^{a}$ being ordinary coordinates, $\theta^{a}$ Grassmann coordinates, $a \in\{0,1, \ldots, d\}, \tau$ an ordinary time parameter and $\xi$ an anticommuting time parameter and assuming $X^{a}=x^{a}$ $+\epsilon \xi \theta^{a}$ as a supercoordinate ${ }^{4}$ and making a choice for $\epsilon$ ) about which we shall not go into detail here, the $\widetilde{\widetilde{a}}^{a}$ appear to be zero as one of the constraints. This constraint has been used to put $\widetilde{\widetilde{a}}^{a}$ 's equal to zero in the further calculations in this reference and it was used as argument for dropping the $\widetilde{\widetilde{S}}^{a b}$ part of the Lorentz generator $\mathcal{S}^{a b} .^{5}$ Let us stress that once the $\widetilde{a}^{a}$ or $d x^{a} \approx \widetilde{\nabla}$ is dropped and accordingly the $\widetilde{\widetilde{S}}^{a b}$ is dropped-for whatever reason-one is no longer asking for the representation under the same Lorentz transformations (including rotations) and one shall not expect to find say integer spin even if the field considered is purely constructed from scalars, vectors, and tensors.

\footnotetext{
${ }^{4}$ The supercoordinate, depending on ordinary $\left(x^{a}\right)$ and Grassmann coordinates $\left(\theta^{a}\right)$ demonstrates the common Lorentz transformations.

${ }^{5}$ We point out in Ref. [2] that this constraint, once taken into account by being put to zero in all the physical operators, was not further treated as a weak equation. Furthermore, such a weak equation- $\widetilde{\widetilde{a}}^{a}$ is an odd Grassmann operator-cannot at all be fulfilled.
}

Let us point out further that what happens is that either the $\theta^{a}$ polynomials of in the one of us approach or the linear combinations of $p$ forms in the Kähler approach, can be formulated as double spinors, i.e., expressions with two (Dirac) spinor indices, $\alpha$ and $\beta$ say, and that the "cheat" consists of dropping from the concept of Lorentz transformations the transformations in one of these indices. In fact we can rewrite the following.

For the even $d$ case one has either

$$
\psi_{\alpha \beta}\left(\left\{\theta^{a}\right\}\right):=\sum_{i=0}^{d}\left(\gamma_{a_{1}} \gamma_{a_{2}} \cdots \gamma_{a_{i}}\right)_{\alpha \beta} \theta^{a_{1}} \theta^{a_{2}} \cdots \theta^{a_{i}}
$$

or

$$
\begin{aligned}
\psi_{\alpha \beta}\left(\left\{d x^{a}\right\}\right):= & \sum_{i=0}^{d}\left(\gamma_{a_{1}} \gamma_{a_{2}} \cdots \gamma_{a_{i}}\right)_{\alpha \beta} \\
& \times d x^{a_{1}} \bigwedge d x^{a_{2}} \wedge \cdots d x^{a_{i}} \wedge
\end{aligned}
$$

while for the odd $d$ case one has either

$$
\psi_{\alpha \beta \Gamma}\left(\left\{\theta^{a}\right\}\right):=\sum_{i=0}^{d}\left(\gamma_{(\Gamma) a_{1}} \gamma_{(\Gamma) a_{2}} \cdots \gamma_{(\Gamma) a_{i}}\right)_{\alpha \beta} \theta^{a_{1}} \theta^{a_{2}} \cdots \theta^{a_{i}}
$$

or

$$
\begin{aligned}
\psi_{\alpha \beta \Gamma}\left(\left\{\theta^{a}\right\}\right):= & \sum_{i=0}^{d}\left(\gamma_{(\Gamma) a_{1}} \gamma_{(\Gamma) a_{2}} \cdots \gamma_{(\Gamma) a_{i}}\right)_{\alpha \beta} \\
& \times d x^{a_{1}} \bigwedge d x^{a_{2}} \wedge \cdots d x^{a_{i}} \wedge
\end{aligned}
$$

with the convention $a_{1}<a_{2}<a_{3}<\cdots<a_{i}$. Here the sums run over the number $i$ of factors in the products of $d x^{a} \wedge$ or $\theta^{a}$ coordinates, a number which is the same as the number of gamma-matrix factors. It should be remarked that we include the possibility $i=0$, which means no factors, and is taken to mean that the product of zero $d x^{a} \wedge$ or $\theta^{a}$ factors is unity and the product of zero gamma matrices is the unit matrix. The indices $\alpha, \beta$ are the spinor indices and taking the product of gamma matrices conceived of as matrices the symbol $(\cdots)_{\alpha \beta}$ stands for an element in the $\alpha$ th row and in the $\beta$ th column. There is an understood Einstein convention summation over the contracted vector indices $a_{k}, k=1,2, \ldots, i$. The gamma matrices are, in the even-dimension case, $2^{d / 2}$ by $2^{d / 2}$ matrices and in the odd-dimension case, $2^{(d-1) / 2}$ by $2^{(d-1) / 2}$ matrices. In the odd case we have worked with two (slightly) different gamma-matrix choices-and thus have written the gamma matrices as depending on the $\operatorname{sign}^{6} \Gamma$ as $\gamma(\Gamma)_{a_{k}}$-namely gamma matrix choices obeying

$$
\Gamma=\gamma_{1} \gamma_{2} \cdots \gamma_{d}
$$

\footnotetext{
${ }^{6} \mathrm{We}$ use the same symbol for the matrix $\Gamma=\gamma_{1} \gamma_{2} \cdots \gamma_{d}$ and for the sign, pointing out that the $\gamma$ matrices depend on the value of $\Gamma$, which is \pm 1 .
} 
The $\gamma_{a}$ matrices should, of course, be constructed so that they obey the Clifford algebra

$$
\left\{\gamma_{a}, \gamma_{b}\right\}=2 \eta^{a b}
$$

and we could, e.g., choose

$$
\begin{aligned}
\gamma_{1} & :=i \sigma_{2}^{1} \times \sigma_{3}^{2} \times \sigma_{3}^{3} \times \cdots \times \sigma_{3}^{n}, \\
\gamma_{2} & :=-i \sigma_{1}^{1} \times \sigma_{3}^{2} \times \sigma_{3}^{3} \times \cdots \times \sigma_{3}^{n}, \\
\gamma_{3} & :=i I^{1} \times \sigma_{2}^{2} \times \sigma_{3}^{3} \times \cdots \times \sigma_{3}^{n}, \\
\gamma_{4} & :=i I^{1} \times(-) \sigma_{1}^{2} \times \sigma_{3}^{3} \times \cdots \times \sigma_{3}^{n}, \\
\gamma_{5} & :=i I^{1} \times I^{2} \times \sigma_{2}^{3} \times \cdots \times \sigma_{3}^{n}, \\
& \vdots \quad \vdots \quad \vdots \quad \vdots \quad \ddots \quad \vdots, \\
\gamma_{2 n-1} & :=i I^{1} \times I^{2} \times I^{3} \times \cdots \times \sigma_{2}^{n}, \\
\gamma_{2 n} & :=i I^{1} \times I^{2} \times I^{3} \times \cdots \times(-) \sigma_{1}^{n},
\end{aligned}
$$

for an even dimension $d=2 n$, while for an odd dimension $d=2 n+1$ the gamma matrix $\gamma^{2 n+1}$ has to be included:

$$
\gamma_{2 n+1}:=i \Gamma \sigma_{3}^{1} \times \sigma_{3}^{2} \times \sigma_{3}^{3} \times \cdots \times \sigma_{3}^{n},
$$

from where it follows that $\Gamma=\Pi_{a}^{2 n+1} \gamma_{a}$. (The choice of phases of Ref. [10] are achieved by using the minus signs in parentheses.) The above metric is supposed to be Euclidean, with $\delta^{a b}=-\eta^{a b}$. For the Minkowski metric $\gamma_{2 n} \rightarrow-i \gamma_{2 n}$ has to be taken, if the index $2 n$ is recognized as the "time" index. We shall make use of the Minkowski metric, counting the $\gamma^{a}$ from $0,1,2,3,5, \ldots, d$, and assuming the metric $\eta^{a b}$ $=\operatorname{diag}(1,-1,-1, \ldots,-1)$.

In this notation we can see that for fixed values of the index $\beta$ we obtain one of the four bispinors in Table I, conceived of as a spinor in the index $\alpha$ and with the understanding that the $\widetilde{a}^{a}$ in the table lead to the corresponding $\theta^{a}$, when acting on the vacuum state. The equivalent table for the Kähler approach follows by replacing $\theta^{a}$ by $d x^{a} \wedge$.

It is our main point to show that the action by the operators $d x^{a} \bigvee$ or $\widetilde{a}^{a}$ and $d x^{a} \widetilde{\widetilde{V}}$ or $\widetilde{a}^{a}$ in the representation based on the basis $\psi_{\alpha \beta}\left(\left\{d x^{a}\right\}\right)$ or $\psi_{\alpha \beta}\left(\left\{\theta^{a}\right\}\right)$ with

$$
\alpha, \beta \in\left(1,2, \ldots,\left\{\begin{array}{rc}
2^{(d-1) / 2} & \text { for } d \text { odd } \\
2^{d / 2} & \text { for } d \text { even }
\end{array}\right\}\right),
$$

defined by Eq. (48) or (49), transforms the index $\alpha$ and $\beta$, respectively, of the basis $\psi_{\alpha \beta}\left(\left\{d x^{a}\right\}\right)$ or equivalently $\psi_{\alpha \beta}\left(\left\{\theta^{a}\right\}\right)$ as follows:

$$
\text { either } d x^{a} \widetilde{\vee} \psi_{\alpha \beta(\Gamma)}\left(\left\{d x^{a}\right\}\right) \propto \gamma_{\alpha \gamma}^{a} \psi_{\gamma \beta(\Gamma)}\left(\left\{d x^{a}\right\}\right),
$$

corresponding to $\widetilde{a}^{a} \psi_{\alpha \beta(\Gamma)}\left(\left\{\theta^{a}\right\}\right) \propto \gamma_{\alpha \gamma}^{a} \psi_{\gamma \beta(\Gamma)}\left(\left\{\theta^{a}\right\}\right)$,

$$
\text { or } d x^{a} \widetilde{\widetilde{V}} \psi_{\alpha \beta(\Gamma)}\left(\left\{d x^{a}\right\}\right) \propto \psi_{\alpha \gamma(-\Gamma)}\left(\left\{d x^{a}\right\}\right) \gamma_{\gamma \beta}^{a} \text {, }
$$

corresponding to $\widetilde{a}^{a} \psi_{\alpha \beta(\Gamma)}\left(\left\{\theta^{a}\right\}\right) \propto \psi_{\alpha \gamma(-\Gamma)}\left(\left\{\theta^{a}\right\}\right) \gamma_{\gamma \beta}^{a}$, which demonstrates the similarities between the spinors of the approach of one of us, and the Kähler approach: the operators $d x^{a} \tilde{V}$ and $\widetilde{a}^{a}$ transform the left index of the basis $\psi_{\alpha \beta(\Gamma)}\left(\left\{d x^{a}\right\}\right), \quad$ or correspondingly of the basis $\psi_{\alpha \beta(\Gamma)}\left(\left\{\theta^{a}\right\}\right)$, while keeping the right index fixed and the operators $d x^{a} \widetilde{\widetilde{V}}$ and $\widetilde{\widetilde{a}}^{a}$ transform the right index of the basis $\psi_{\alpha \beta(\Gamma)}\left(\left\{d x^{a}\right\}\right)$, or correspondingly of the basis $\psi_{\alpha \beta(\Gamma)}\left(\left\{\theta^{a}\right\}\right)$ and keep the left index fixed. Under the action of either $d x^{a} \widetilde{V}$ and $\widetilde{a}^{a}$ or $d x^{a} \widetilde{\widetilde{V}}$ and $\widetilde{\widetilde{a}}^{a}$ the basic functions transform as spinors. The index in parentheses $(\Gamma)$ is defined only for odd $d$. We can count that the number of spinors is $2^{d}$ in either the Mankoč approach or in the Kähler approach; the $d$-dimensional Grassmann space or the space of $p$ forms has $2^{d}$ basic functions.

We shall prove the above formulas for the action of the $\widetilde{a}^{a}$ and $\widetilde{\widetilde{a}}^{a}$. The proof is also valid for the Kähler case if $\widetilde{a}^{a}$ is replaced by $d x^{a} \widetilde{V}$ and $\widetilde{a}^{a}$ by $d x^{a} \widetilde{\widetilde{V}}$.

Proof of our formula for the action of $\widetilde{a}^{a}$ and $\widetilde{a}^{a}$. Let us first introduce the notation

$$
\gamma^{A}:=\gamma^{a} \gamma^{b} \cdots \gamma^{c}, \quad \gamma^{\bar{A}}:=\gamma^{c} \gamma^{b} \cdots \gamma^{a},
$$

with $a<b<\cdots<c \in A$. We recognize that

$$
\operatorname{Tr}\left(\gamma_{A} \gamma^{\bar{B}}\right)=\operatorname{Tr}(\mathrm{I}) \delta_{A}{ }^{B}, \quad \sum_{A}\left(\gamma_{A}\right)_{\alpha \beta}\left(\gamma^{\bar{A}}\right)_{\gamma \delta}=2^{d} \delta_{\alpha \gamma} \delta_{\beta \delta},
$$

and

$$
\begin{aligned}
& \sum_{A}\left(\gamma_{A}\right)_{\alpha \beta}\left(\gamma^{c} \gamma^{\bar{A}}\right)_{\gamma \delta}=2^{d}\left(\gamma^{c}\right)_{\gamma \alpha} \delta_{\beta \delta}, \\
& \sum_{A}\left(\gamma_{A}\right)_{\alpha \beta}\left(\gamma^{\bar{A}} \gamma^{c}\right)_{\gamma \delta}=2^{d}\left(\gamma^{c}\right)_{\beta \delta} \delta_{\alpha \gamma},
\end{aligned}
$$

with $a_{1}<a_{2}, \ldots,<a_{i} \in A$ in ascending order and with $\bar{A}$ in descending order. The above formulas are true only for the Euclidean signature $4 \eta_{a b}=(1,1,1, \ldots, 1)$ and would be modified in the Minkowski case. [The first of the formulas of Eq. (54) is trivially true. The second one might be proven by noticing that its contraction with $\gamma^{\bar{B}}$ in the indices $\alpha$ and $\beta$ and with $\gamma^{\bar{C}}$ in the indices $\gamma$ and $\delta$ simultaneously, would be the same on both sides of the equation. By arguing that all these "traces" are sufficient to specify uniquely such four index objects, the equation is derived.] Using the first equation we find

$$
\theta^{A}=\frac{1}{2^{d}}\left(\gamma^{\bar{A}}\right)_{\alpha \beta} \psi_{\beta \alpha(\Gamma)}\left(\left\{\theta^{a}\right\}\right) .
$$

The index $(\Gamma)$ has meaning only for an odd $d$, which is why we put it in parentheses. We may accordingly write

$$
\psi_{\alpha \beta(\Gamma)}\left(\left\{\theta^{a}\right\}\right):=\sum_{A} \frac{1}{2^{d}}\left(\gamma_{A}\right)_{\alpha \beta}\left(\gamma^{\bar{A}}\right)_{\gamma \delta} \psi_{\delta \gamma(\Gamma)}\left(\left\{\theta^{a}\right\}\right) .
$$


Then we find, taking into account that $\widetilde{a}^{a}|\rangle=\theta^{a}, \widetilde{a}^{a}|\rangle$ $=-i \theta^{a}$, where |\rangle is a vacuum state [which means that $\psi\left(\left\{\theta^{a}\right\}\right)=|\rangle$ for $\left.\psi\left(\left\{\theta^{a}\right\}\right)=1\right]$, and Eq. (7)

$$
\begin{aligned}
\widetilde{a}^{c} \psi_{\alpha \beta(\Gamma)}\left(\left\{\theta^{a}\right\}\right) & :=\sum_{A}\left(\gamma_{A}\right)_{\alpha \beta} \widetilde{a}^{c} \theta^{A}=\sum_{A}\left(\gamma_{A}\right)_{\alpha \beta} \widetilde{a}^{c} \widetilde{a}^{A}|\rangle \\
& =\sum_{A} \frac{1}{2^{d}}\left(\gamma_{A}\right)_{\alpha \beta}\left(\gamma^{c} \gamma^{\bar{A}}\right)_{\gamma \delta} \psi_{\delta \gamma(\Gamma)}\left(\left\{\theta^{a}\right\}\right) .
\end{aligned}
$$

Consequently using the above relations starting from Eq. (55) we further find

$$
\widetilde{a}^{c} \psi_{\alpha \beta(\Gamma)}\left(\left\{\theta^{a}\right\}\right):=\left(\gamma^{c}\right)_{\alpha \gamma} \psi_{\gamma \beta(\Gamma)}\left(\left\{\theta^{a}\right\}\right) .
$$

We find in a similar way

$$
\begin{aligned}
\widetilde{\widetilde{a}}^{c} \psi_{\alpha \beta(\Gamma)}\left(\left\{\theta^{a}\right\}\right) & :=\sum_{A}\left(\gamma_{A}\right)_{\alpha \beta} \widetilde{\widetilde{a}}^{c} \widetilde{a}^{A}|\rangle=\sum_{A}\left(\gamma_{A}\right)_{\alpha \beta} \widetilde{a}^{A} \widetilde{\widetilde{a}}^{c}|\rangle \\
& =\sum_{A}\left(\gamma_{A}\right)_{\alpha \beta} \widetilde{a}^{A} \widetilde{\widetilde{a}}^{c}|\rangle \\
& =\sum_{A} \frac{1}{2^{d}}\left(\gamma_{A}\right)_{\alpha \beta}\left(\gamma^{\bar{A}} \gamma^{c}\right)_{\gamma \delta} \psi_{\delta \gamma(\Gamma)}\left(\left\{\theta^{a}\right\}\right),
\end{aligned}
$$

which finally gives

$$
\widetilde{\widetilde{a}}^{c} \psi_{\alpha \beta(\Gamma)}\left(\left\{\theta^{a}\right\}\right):=\psi_{\alpha \gamma(-\Gamma)}\left(\left\{\theta^{a}\right\}\right)\left(\gamma^{c}\right)_{\gamma \beta}
$$

We have therefore proved the two equations which determine the action of the operators $\widetilde{a}^{a}$ and $\widetilde{\widetilde{a}}^{a}$ on the basic function $\psi_{\alpha \gamma(-\Gamma)}\left(\left\{\theta^{a}\right\}\right)$.

\section{GETTING AN EVEN GAMMA MATRIX}

According to Eqs. (58),(59) it is obvious that the $\gamma^{a}$ matrices, entering into the Dirac-Kähler approach or the approach of one of us for spinors, have an odd Grassmann character since both, $d x^{a} \tilde{\nabla}$ and $\widetilde{a}^{a}$ as well as $d x^{a} \widetilde{\widetilde{V}}$ and $\widetilde{\widetilde{a}}^{a}$ have an odd Grassmann character. They therefore transform a Grassmann odd basic function into a Grassmann even basic function changing fermion fields into boson fields. It is clear that such $\gamma^{a}$ matrices are not appropriate to enter into the equations of motion and Lagrangians for spinors.

There are several possible ways to avoid this trouble [2]. One of them was presented in Sec. IV. If working with $d x^{a} \tilde{\nabla}$ or $\widetilde{a}^{a}$ alone, putting $d x^{a} \approx$ or $\widetilde{\widetilde{a}}^{a}$ in the Hamiltonian, Lagrangian and all the operators equal to zero, the $\tilde{\gamma}^{a}$ matrices of an even Grassmann character can be defined as proposed in Eq. (27): $\tilde{\gamma}^{a}:=i d x^{0} \widetilde{\widetilde{V}} d x^{a} \widetilde{\vee}$ or $\tilde{\gamma}^{a}:=i \tilde{\widetilde{a}}^{0} \widetilde{a}^{a}$, which fulfill the Clifford algebra $\left\{\tilde{\gamma}^{a}, \tilde{\gamma}^{b}\right\}=2 \eta^{a b}$, while, as we have already said $\widetilde{S}^{a b}=-i / 4\left[\tilde{\gamma}^{a}, \tilde{\gamma}^{b}\right]$. We then have

$$
\tilde{\gamma}^{a} \psi_{\alpha \beta(\Gamma)}\left(\left\{\theta^{a}\right\}\right)=\gamma_{\alpha \gamma}^{a} \psi_{\gamma \delta(-\Gamma)}\left(\left\{\theta^{a}\right\}\right) \gamma_{\delta \beta}^{0}
$$

One can check that $\tilde{\gamma}^{a}$ have all the properties of the Dirac $\gamma^{a}$ matrices. (Replacing $d x^{a} \widetilde{\vee}$ or $\widetilde{a}^{a}$ by $d x^{a} \approx \widetilde{\nabla}$ or $\widetilde{a}^{a}$, respectively, the gamma matrices defined as $\widetilde{\widetilde{\gamma}}^{a}:=$ either $i d x^{0}$ $\tilde{\nabla} d x{ }^{a} \approx$ or $i \widetilde{a}^{0} \widetilde{\widetilde{a}}^{a}$ again have all the properties of the Dirac $\gamma^{a}$ matrices.)

\section{DISCRETE SYMMETRIES}

We shall comment in this section on the discrete symmetries of spinors and vectors in the Hilbert space spanned over either the Grassmann coordinate space or the space of differential forms from the point of view of the one-particle states of massless Dirac (that is the Weyl) particles. In order to define the discrete symmetries of the Lorentz group we introduce the space inversion $P$ and the time inversion $T$ operators in ordinary space-time in the usual way. We shall assume the case $d=4$ :

$$
P x^{a} P^{-1}=x_{a}, \quad T x^{a} T^{-1}=-x_{a}
$$

with the metric $\eta^{a b}, x^{a}=\eta^{a b} x_{b}$ already defined in Sec. II. Since one wants the time reversal operator to leave $p^{0}$, that is the zero component of the ordinary space-time momentum operator $\left(p^{a}\right)$, unchanged $\left(p^{0} \rightarrow p^{0}\right)$, while the space component $\vec{p}$ should change sign $(\vec{p} \rightarrow-\vec{p})$, one also requires

$$
T i T^{-1}=-i \text { leading to } T p^{a} T^{-1}=p_{a} .
$$

We shall first treat spinors. Having the representation of spinors expressed in terms of polynomials of $\theta^{a}$ 's in Table I, which also represents the corresponding superpositions of $p$ forms if $\theta^{a}$ is accordingly substituted by $d x^{a} \wedge$, we expect each of the four copies of Dirac massless spinors to transform under discrete symmetries of the Lorentz transformations in the usual way.

The parity operator $P$ should transform left-handed spinors with $\left\langle\Gamma^{(4)}\right\rangle=-1$ to right-handed spinors with $\left\langle\Gamma^{(4)}\right\rangle=1$, without changing the spin of the spinors. This is what $\tilde{\gamma}^{0}$ [Eq. (27)] does for any of the four copies of the Dirac massless spinors, which are the Weyl bispinors of Table I, separately.

The time reversal operator $T$ should transform left-handed spinors with $\left\langle\Gamma^{(4)}\right\rangle=-1$ and spin $\frac{1}{2}$ to left-handed spinors with $\left\langle\Gamma^{(4)}\right\rangle=-1$ and spin $-\frac{1}{2}$, and that is what the operator

$$
\begin{aligned}
& T=i \tau_{\text {int }} \tau_{x} K, \quad \tau_{\text {int }}=\tilde{\gamma}^{1} \tilde{\gamma}^{3}, \\
& \tau_{x}=\operatorname{diag}(-1,1,1,1), \text { and } K i K^{-1}=-i
\end{aligned}
$$

(where $K$ does not effect the basic spin-states any longer due to the appropriate choice of the basis) does when applied to any of the four copies of the Dirac spinors of Table I. This transformation involves only members of the same copy of the Dirac bispinor. The operators $\tilde{\gamma}^{a}$ which are defined in Eq. (27) have, due to the appropriate choice of phases of the spinors of Table I, the usual chiral matrix representation (for both approaches - the Kähler one and the Mankoč one). 
One would, however, expect that the time and the space reversal operators should work in both spaces-that is, in the ordinary space-time and in the space of either Grassmann polynomials or in the space of $p$ forms-in an equivalent way

$$
\begin{gathered}
P^{\prime} x^{a} P^{\prime-1}=x_{a}, T^{\prime} x^{a} T^{\prime-1}=-x_{a}, \\
P^{\prime} \theta^{a} P^{\prime-1}=\theta_{a} \text { or correspondingly } P^{\prime} d x^{a} \wedge P^{\prime-1}=d x_{a} \wedge, \\
\text { and } T^{\prime} \theta^{a} T^{\prime-1}=-\theta_{a} \\
\text { or correspondingly } T^{\prime} d x^{a} \wedge T^{\prime-1}=-d x_{a} \wedge, \\
T^{\prime} i T^{\prime-1}=-i \text { leading to } T^{\prime} p^{a} T^{\prime-1}=p_{a}
\end{gathered}
$$

and changing equivalently the momenta conjugate to coordinates in either the Kähler approach or that of one of us.
Applying the transformation $P^{\prime}$ of Eq. (64) to any of the four copies of the Dirac bispinors of Table I, one obtains the same result as in the above, that is, the standard definition of the space-reversal operation: $P^{\prime}=P$. Applying the transformation $T^{\prime}$ of Eq. (64) to, let us say, the first spinor of the first copy of the Dirac bispinors of Table I [that is, on $\left.{ }^{1} \widetilde{\psi}_{1}(\{\theta\})\right]$, one obtains the last spinor of the one before the last copy [that is, $\left.{ }^{7} \widetilde{\psi}_{2}(\{\theta\})\right]$. The left-handed spinor with spin $\frac{1}{2}$ transformed to the left-handed spinor of spin $-\frac{1}{2}$, just as it did under the usual time-reversal transformation, except that in this case the copy of spinors has been changed.

One can write down the matrix representation for this second kind of the time-reversal transformation. If we choose for the basis the first copy of bispinors of Table I and the fourth copy of bispinors of Table I, we obtain the matrix

$$
T^{\prime}=\left(\begin{array}{cccc}
0 & 0 & i \sigma_{2} e^{i \varphi} K_{\varphi} & 0 \\
0 & 0 & 0 & i \sigma_{2} e^{i \varphi} K_{\varphi} \\
-i \sigma_{2} e^{-i \varphi} K_{\varphi} & 0 & 0 & 0 \\
0 & -i \sigma_{2} e^{-i \varphi} K_{\varphi} & 0 & 0
\end{array}\right)
$$

where $e^{i \varphi}=1$, due to the choice of the phases of spinors of Table I, and $K_{\varphi}$ means that the complex conjugation has to be performed on the phase coefficients only in an expansion on the basis of Table I. Polynomials of theta have been, due to the choice of the basis, replaced by real spinor state vectors. Let us point out that while the choice of $\tilde{\gamma}^{0}$ [Eq. (27)] enables the two parity operators, the $\tilde{\gamma}^{0}$ one and the $\vec{\theta} \rightarrow$ $-\vec{\theta}$, to become equivalent, it is no freedom left to do the same with the two time operators $T$ and $T^{\prime}$. We can, however, express the time operator $T^{\prime}$ in terms of $K$ and $\tilde{\gamma}^{0}$, if $K$ transforms $i$ into $-i$ also in the $\theta$ part of space: $T^{\prime}=\tilde{\gamma}^{0} K$. This $T^{\prime}$ is the time-reversal operation discussed by Weinberg [6] in Appendix C of his book. ${ }^{7}$

When vectors and scalars are treated in a similar way for either of the two approaches (the polynomial representations in terms of $\theta$ 's can be found in Refs. [2,3,15]), it turns out that the time-reversal operator of Eq. (64) does not transform one copy into another one.

We pay attention in this section only to spin degrees of freedom. The complex conjugation affects, of course, the

\footnotetext{
${ }^{7}$ The two kinds of time reversal operator have already been discussed in Ref. [2]. The appearance of the second kind of time reversal operator in Weinberg's book as well as in Wigner's book [11] was pointed out [12] to the authors at the Workshop "What Comes Beyond the Standard Model"' at Bled (1999), when it was suggested that the second kind could generate states, which may be used to describe the sterile neutrinos.
}

higher part of the internal space as well, affecting the charges of spinors, vectors and tensors, if one thinks of the extension [2] as discussed in Sec. IX.

\section{UNAVOIDABILITY OF FAMILIES}

We want to look at the unusual shift of the spin compared to the a priori spin for a field by replacing a priori generators $M^{a b}=L^{a b}+\mathcal{S}^{a b}$ by another set $\tilde{M}^{a b}=L^{a b}+\widetilde{S}^{a b}$ as a general nice idea. A prerequisite for that working is that the difference between the two proposals for Lorentz generators

$$
\widetilde{\tilde{M}}^{a b}:=M^{a b}-\widetilde{M}^{a b}
$$

is also a conserved set of quantities. In the notation above of course we find

$$
\widetilde{\widetilde{M}}^{a b}=\widetilde{\widetilde{S}}^{a b}
$$

Assuming that there are indeed such two Lorentz generator symmetries in a model, we can ask for the representation under both for a given set of fields, and we can even ask for representation under the difference algebra $\widetilde{\widetilde{M}}^{a b}$. In order to shift from integer spin to half-integer spin in going from $M^{a b}$ to $\widetilde{M}^{a b}$, the representation for the fields in question must at least be spin-1/2 for $\widetilde{\widetilde{M}}^{a b}$. Actually in the cases we discussed the $\widetilde{\widetilde{M}}^{a b}$ were in the Dirac spinor representation. But that means that the representation of the fields which shift representation going from $M^{a b}$ to $\widetilde{M}^{a b}$ have to belong under $\widetilde{M}^{a b}$ 
to at least a spin-1/2, i.e., at least the Weyl spin representation of the Lorentz group, and that has $2^{(d / 2-1)}$ dimensions. But that means then that a given representation of the final $\widetilde{M}^{a b}$ Lorentz group must always occur in at least $2^{(d / 2-1)}$ families (whenever this trick of spin change is used).

\section{GENERALIZATION TO EXTRA DIMENSIONS}

We have discussed the connection between the Grassmann $\theta^{a}$ formulation and the Kähler formalism for general dimension $d$ and thus we could apply it simply in the $d=4$ case, or we could use it in extended models with extra dimensions. One should note that the connection between the spinor and the forms is such that for each extra two dimensions the number of components of a Dirac spinor goes up by a factor of 2, and at the same time the number of families also doubles. This agrees with the fact that adding one extra $\theta^{a}$ doubles the number of terms in the $\theta^{a}$ polynomials, and thus adding two would make this number four times as big.

Let us now study the application of the extra degrees of freedom, which consists of, let us suppose the Grassmann $\theta^{a}$ s or the Kähler differential forms, to the case where the $d$-dimensional space is used in a Kaluza-Klein type model: that is to say, we look here at a Kaluza-Klein model extended with $\theta^{a}$ s or the forms, much more rich than usual Kaluza-Klein. It has long been suggested [2] that special kinds of rotations of the spins in the extra $(d-4)$ dimensions manifest themselves as generators for charges observable at the end for the four-dimensional particles. It is the highlight and main reason for Ref. [2] that since both the extra dimension spin degrees of freedom and the ordinary spin degrees of freedom originate from the $\theta^{a}$ 's or the forms we have a unification of these internal degrees of freedom. We can say then that the generators rotating these degrees of freedom, namely, the just mentioned charges acting as higher dimensional spins (at high energy) and the four-dimensional spin, are unified.

Such rotations of the internal spin degrees of freedom, in order to correspond to a Kaluza-Klein gauge field with massless gauge bosons, would have to represent full symmetries of the vacuum state, i.e., they should correspond to Killingvectors, as in usual Kaluza-Klein but with the further degrees of freedom also corresponding to symmetry for the latter. So at the end we may consider also the charges associated with the internal spin as ordinary Kaluza-Klein charges, of course in the sense of the very rich model considered here. But of course unless we have the $\theta^{a}$ or forms degrees of freedom one could risk that the gauge field from such symmetry could be practically decoupled.

Let us now look at what the "families" found in the Grassmann or Dirac-Kähler approach will develop into in case we use it for a Kaluza-Klein type model, as just proposed. Usually the number of surviving massless fermions in the $(3+1)$ space consists only of those which are connected with "zero-modes." This is to be understood as follows: we use the Atiyah-Singer theorem to ensure that in $(d-4)$-dimensional space-the compactified part of the space-some modes are zero modes. Correspondingly, each such zero mode enables the existence of zero mass particles in the $(3+1)$-dimensional (flat) space-time.

If the model had a strength for the compact space AtiyahSinger (AS) theorem "AS strength" and if the dimension of the full space, the number of $\theta^{a}$ 's, is $d$, so that the number of families at the $d$-dimensional level becomes $2^{d / 2}$, the total number of "families" observable at low energy should be

$$
\text { No. families = "AS strength" } \times 2 d / 2 \text {. }
$$

As an example take the model $[2,14]$ which has $d=14$ and at first—at the high-energy level- $\mathrm{SO}(1,13)$ Lorentz group, but which should be broken (in two steps) first to $\mathrm{SO}(1,7) \times \mathrm{SO}(6)$ and then to $\mathrm{SO}(1,3) \times \mathrm{SU}(3) \times \mathrm{SU}(2)$. For this example the number of families would come in multiples of $2^{7}$.

\section{DISCUSSION OF SPECIES DOUBLING PROBLEM}

We may see the appearance of equally many (namely, $2^{d / 2-1}$ ) right-handed and left-handed "flavors" in the Kähler model as an expression for the no-go theorem [7] for putting chiral charge-conserving fermions on the lattice, insofar as we could make attempts to make lattice fermions along the lines of Becher and Joos [9] it would of course have been a counterexample to the no-go [7] theorem if there had been a different number of right and of left Weyl particle species in the Becher-Joos model, because in the free model the number of particles functions as a conserved charge. As is very well known the Becher-Joos model is really just the Kogut-Susskind [13] lattice fermion model; it is also well known that it does not violate the no-go theorem [7] and this is because there is this species doubling, which can be interpreted as the flavors. Becher and Joos show that the KogutSusskind lattice description of Dirac fields is equivalent to the lattice approximation of the Dirac-Kähler equation (see page 344 in the Becher-Joos [9] article).

This Kogut-Susskind model is one that gives us Dirac particles, but we can seek to get to Weyl particles in a naive $\Gamma^{(4)}$ (or $\widetilde{\Gamma}^{(4)}$ or $\gamma^{5}$ in the usual notation) projecting way, but of course now such a projection would have to be translated into the language with the vector and scalar fields in Kähler formulation, and it is rather easy to see [15] that requiring only one $\Gamma^{(4)}$ projection implies that the coefficient to one $p$ form, say $d x^{A}, d x^{A}=d x^{a_{1}} \wedge d x^{a_{2}} \wedge \ldots \wedge d x^{a_{i}}$, should relate (just by a sign $\times i$ ) to that of the $* d x^{A}$ associated by the Hodge star $(*)$. Actually, we easily see that requiring the restriction that

$$
\left(1+\Gamma^{(4)}\right) \psi=0
$$

in the language of Kähler becomes

$$
\left(1+i^{*}\right) u=0
$$

If, as Joos and Becher did, we want to put the theory on the lattice there is a difficulty in just imposing this constraint, because the natural relation imposed by the Hodge star on the lattice would go from the lattice to the dual lattice, and 
we could not identify without a somewhat ambiguous choice the $*$ dual of a given lattice element, so as to impose the "self-duality" condition.

Could we possibly invent a way to circumvent the no-go theorem [7] for chirality conserving fermions on the lattice by making the species doublers bosons instead of fermions, both having though spin-1/2? In the formulation by one of us which we have related to the Kähler formulation there is (naturally) a different Grassmaniann character assigned to different components of the wave function. In fact the wave function with coefficients to monomial terms that are products of different sets of (mutually different) $\theta^{a}$ variables-in the sense, of course, that a polynomial is given by its coefficients - and thus the coefficients to the products with an even number of factors have a different Grassmannian character from those of the odd number of factors. That actually is, in the theory of one of us, a somewhat embarrassing reason for a superselection rule which, though, may be overcome by taking into account the charges related to extra dimensions appearing in that model. But here we now want to point out the hope that these very Grassmann character problems may be used as a new idea to circumvent the no-go theorem. In fact we could hope that spin-1/2 and, say, left handed flavor appear with fermionic statistics (the Grassmann odd character) while spin-1/2 flavor with bosonic statistics would appear as right handed, and that even on the lattice.

\section{CONCLUDING REMARKS}

The way that Mankoc [2] chooses to quantize the system, that is a particle moving in ordinary and Grassmann coordinate space, is to let the wave function be allowed to be any function of the $d$ Grassmann variables $\theta^{a}$, so that any such function represents a state of the system. But in this quantization the $\widetilde{a}^{a}$ 's cannot be put weakly to zero. In other words that quantization turned out not to obey the equation expected from the expression for the canonical momentum $p^{\theta a}$, being proportional to the coordinate $\theta^{a}$ as derived from the Lagrangian. If, however, in the operators such as the Hamiltonian and the Lorentz transformation operators $\widetilde{\widetilde{a}}^{a}$ 's are just put strongly to zero, so that all the operators only depend on $\widetilde{a}^{a}$, while either $\widetilde{a}^{a}$ or $\widetilde{\widetilde{a}}^{b}$ fulfill the Clifford algebra: $\left\{\widetilde{a}^{a}, \widetilde{a}^{b}\right\}=0$ and $\left\{\widetilde{a}^{a}, \widetilde{a}^{b}\right\}=2 \eta^{a b}=\left\{\widetilde{a}^{a}, \widetilde{a}^{b}\right\}$, the expressions obtained after having put the $\widetilde{a}^{a}$ 's to zero describe spinor degrees of freedom. In particular, only the operators $\widetilde{S}^{a b}$ are used as the Lorentz generator. One has accordingly the new Lorentz transformations instead of the a priori one used in the wave function on Grassmann space quantization. In that case the argument for having only integer spin breaks down, what the calculations indeed confirm to happen.

We should now attempt to get an understanding of what goes on here by using a basis inspired from the Dirac-Kähler construction, which is a way often used on lattices to implement fermions on the lattice. The Dirac-Kähler construction starts from a field theory with a series of fields which are zero form, one form, two form, $\ldots, d$ form. They can be thought of as being expanded on a basis of all the wedge product combinations of the basis $d x^{1}, d x^{2}, \ldots, d x^{d}$ for the one forms, including wedge products from zero factors to $d$ factors. In the Dirac-Kähler construction one succeeds in constructing $2^{d / 2}$ Dirac spinor fields out of these "all types of forms." This construction is impossible without a "cheat" in much the same way as Mankoč's approach ought to be.

We have pointed out clearly in this paper how this "cheat" occurs in both approaches, showing up all the similarities of the two approaches and using the simple presentation of the quantum mechanics in Grassmann space, not to only simplify the Dirac-Kähler approach but also to generalize it. We have shown in particular that in both approaches in addition to the (two kinds of) generators for the Lorentz transformations for spinors also the generators for vectors and tensors exist. There are four copies of the Weyl bispinors. One kind of the spinorial type of the Lorentz transformations defines the Weyl spinors, another kind transforms one copy of Weyl spinors into another of the same Grassmann character. We also have shown the two kinds of the time reversal operators, as well as the fact that in Grassmann space or space of differential forms of $d$ dimensions, $d>4$, spins and charges unify. We pointed out the necessity of defining the gamma matrices of an even Grassmann character.

\section{ACKNOWLEDGMENTS}

This work was supported by Ministry of Science and Technology of Slovenia as well as by funds CHRX-CT-940621, INTAS 93-3316, INTAS-RFBR 95-0567, SCI-0430-C (TSTS).
[1] E. Kähler, Rend. Mat. Ser. V 21, 452 (1962).

[2] N. Mankoč Borštnik, Phys. Lett. B 292, 25 (1992); Nuovo Cimento A 105, 1461 (1992); J. Math. Phys. 34, 3731 (1993); Int. J. Mod. Phys. A 9, 1731 (1994); J. Math. Phys. 36, 1593 (1995); Mod. Phys. Lett. A 10, 587 (1995); hep-th/9406083; N. Mankoč Borštnik and A. Borštnik, J. Phys. G 24, 963 (1998); in Proceedings of the International Conference “'Quantum Systems, New Trends and Methods,'” Minsk, 1994, edited by A. O. Barut, I. D. Feranchuk, Ya. M. Shnir, and L. M. Tomil'chik (World Scientific, Singapore, 1995), p. 312; in
Proceedings of the US-Polish Workshop "Physics from Planck Scale to Electroweak Scale," Warsaw, 1994, edited by P. Nath, T. Taylor, and S. Pokorski (World Scientific, Singapore, 1995), p. 86; Proceedings of the 7th Adriatic Meetings on High Energy Physics, Brijuni, Croatia, 1994, edited by D. Klabučar, I. Picek, and D. Tadić (World Scientific, Singapore, 1995), p. 296; Proceedings of the Barut Memorial Conference on Group Theory in Physics [Tr. J. Phys. 21, 321 (1997)]; Proceedings of the International Workshop on "What Comes Beyond the Standard Model,'” Bled, Slovenia, 1998, edited by N. Mankoč 
Borštnik, H. B. Nielsen, and C. Froggatt (DMFA, Založništvo, 1998), hep-ph/9905357, p. 20; Proceedings of the VIIth International Conference 'Symmetry Methods in Physics,' Dubna, 1995, edited by Sissakian and G. P. Pogosyan, Joint Institute for Nuclear Research Report No. 141980 Dubna.

[3] N. Mankoč Borštnik and S. Fajfer, Nuovo Cimento Soc. Ital. Fis., B 112, 1637 (1997).

[4] N. Mankoč Borštnik and H. Bech Nielsen, hep-th/0001196.

[5] M. Reuter, J. Math. Phys. 40, 5593 (1999).

[6] S. Weinberg, The Quantum Theory of Fields (Cambridge University Press, Cambridge, England, 1995), Vol. I.

[7] H. B. Nielsen and M. Ninomija, Phys. Lett. 105B, 219 (1981); Nucl. Phys. B185, 20 (1981).

[8] P. Di Vecchia and F. Ravndal, Phys. Lett. 73A, 371 (1979).

[9] P. Becher and H. Joos, Z. Phys. C 15, 343 (1982).

[10] H. Georgi, Lie Algebra in Particle Physics, Frontiers in Phys- ics A: Lecture Notes and Reprint Series (Benjamin Cummings, New York, 1982).

[11] E. P. Wigner, in Group Theoretical Concepts and Methods in Elementary Particle Physics, edited by F. Gürsey (Gordon and Breach, New York, 1964).

[12] R. Erdem, Proceedings of the International Workshop "What Comes Beyond the Standard Model,"' Bled, 1999 (unpublished).

[13] J. Kogut and L. Susskind, Phys. Rev. D 13, 1043 (1976).

[14] Mankoč Borštnik and Nielsen [4].

[15] H. B. Nielsen and N. Mankoč Borštnik, in Proceedings of the International Workshop on "What Comes Beyond the Standard Model," Bled, Slovenia, 1998, edited by N. Mankoč Borštnik, H. B. Nielsen, and C. Froggatt (DMFA, Založništvo, 1998), p. 68; Report No. IJS.TP.99/17 or NBI-HE-99-35 or CERN-TH/99-288; hep-ph/9905357; hep-th/9909169. 